\title{
Are radiomics features universally applicable to different organs?
}

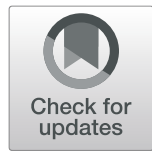

Seung-Hak Lee ${ }^{1,2,3}$, Hwan-ho Cho ${ }^{1,2}$, Junmo Kwon ${ }^{1,2}$, Ho Yun Lee ${ }^{4,5^{*}}$ and Hyunjin Park ${ }^{2,6^{*}}$ (D)

\begin{abstract}
Background: Many studies have successfully identified radiomics features reflecting macroscale tumor features and tumor microenvironment for various organs. There is an increased interest in applying these radiomics features found in a given organ to other organs. Here, we explored whether common radiomics features could be identified over target organs in vastly different environments.

Methods: Four datasets of three organs were analyzed. One radiomics model was constructed from the training set (lungs, $n=401$ ), and was further evaluated in three independent test sets spanning three organs (lungs, $n=59$; kidneys, $n=48$; and brains, $n=43$ ). Intensity histograms derived from the whole organ were compared to establish organ-level differences. We constructed a radiomics score based on selected features using training lung data over the tumor region. A total of 143 features were computed for each tumor. We adopted a feature selection approach that favored stable features, which can also capture survival. The radiomics score was applied to three independent test data from lung, kidney, and brain tumors, and whether the score could be used to separate high- and low-risk groups, was evaluated.
\end{abstract}

Results: Each organ showed a distinct pattern in the histogram and the derived parameters (mean and median) at the organ-level. The radiomics score trained from the lung data of the tumor region included seven features, and the score was only effective in stratifying survival for other lung data, not in other organs such as the kidney and brain. Eliminating the lung-specific feature (2.5 percentile) from the radiomics score led to similar results. There were no common features between training and test sets, but a common category of features (texture category) was identified.

Conclusion: Although the possibility of a generally applicable model cannot be excluded, we suggest that radiomics score models for survival were mostly specific for a given organ; applying them to other organs would require careful consideration of organ-specific properties.

Keywords: Radiomics, Macroscale tumor features, Tumor microenvironment, Computed tomography, Magnetic resonance imaging, Survival analysis

\footnotetext{
* Correspondence: hoyunlee96@gmail.com; hyunjinp@skku.edu

${ }^{4}$ Department of Radiology and Center for Imaging Science, Samsung Medical

Center, Sungkyunkwan University School of Medicine, 81 Irwon-Ro,

Gangnam-Gu, Seoul 06351, South Korea

${ }^{2}$ Center for Neuroscience Imaging Research, Institute for Basic Science (IBS),

Suwon 16419, South Korea

Full list of author information is available at the end of the article
}

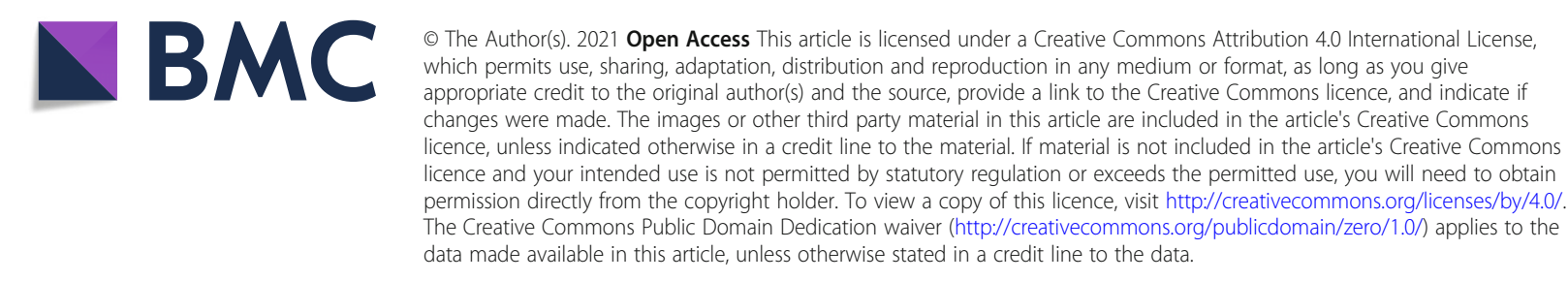




\section{Background}

Modern oncology is moving toward pursuing precision medicine, and medical imaging is an important factor in such an environment [1]. Traditionally, medical imaging has been interpreted by trained expert radiologists using imaging features. A few imaging features (e.g., tumor volume and shape) were used to assess tumor properties of various target organs [2-4]. Recently, medical imaging has been analyzed using a radiomics approach, which considers hundreds or even thousands of mineable features $[5,6]$. With so many available features, radiomics has the potential to provide personalized information for various organs over different diseases $[5,7,8]$. There have been many successful radiomics studies reporting better performance than conventional imaging studies [9-11].

Macroscale features of the tumor such as size, shape, and texture are reflected in radiomics features [5, 12]. These features were useful in quantitively describing various aspects of tumor especially for evaluating regional heterogeneity. The tumor margin is an extremely dynamic area composed of immune cells, rich vasculature, lymphatics, fibroblasts, pericytes, and adipocytes, related to the tumor microenvironment [13-15]. It plays a vital role in prognosis and therapy response, especially for immunotherapy $[16,17]$. Hence, the radiomics features in the marginal region might reflect the tumor microenvironment. Radiomics studies have identified essential features that non-invasively capture information related to the tumor microenvironment. In particular, quantitative imaging features based on histogram, texture, and shape provide information about the tumor microenvironment $[5,18]$. One study showed that homogeneity, dissimilarity, contrast, and energy from the texture category of features reflected the local immune microenvironment of non-small cell lung cancer (NSCLC) [15]. Another study identified homogeneity and difference entropy from the texture category as biomarkers that could predict immune response [19]. The identified radiomics features are largely specific to the organ being modeled, the associated macroscale features and tumor microenvironment, imaging modality, and acquisition parameters. Still, many studies evaluated models trained in one setting to another setting possibly in different organs $[17,18]$. This is because tumors in different organs might share common properties. Thus, there is a scientific curiosity in applying radiomics features found in a given organ to other tumors in different organs [20, 21]. A seminal study by Aerts et al. explored the application of the same radiomics features to two different organs, but this topic was not their primary aim and was thus insufficiently explored. A seminal study explored the application of the same radiomics features to two different organs (i.e., lung and head \& neck cancers) and was successful at classifying the risk groups [20]. Furthermore, the study had issues regarding how the features were selected. A recent study explored the feasibility of applying the same set of radiomics features to intrahepatic cholangiocarcinoma, osteosarcoma, and pancreatic neuroendocrine tumors [21]. This translates to finding a "universal" set of radiomics features that can be applied to potentially different tumor microenvironments, in which they succeeded. Therefore, there is a need to investigate this issue further.

To this end, our study aimed to explore whether common radiomics features can be identified across different target organs. We evaluated the radiomics features of tumors in three organs (i.e., lungs, kidneys, and brains). We trained a radiomics model and chose features using lung data for survival analysis. The chosen features were applied to three independent test datasets of lung, kidney, and brain tumors, and the possibility of using the features to separate high- and low-risk groups was explored.

\section{Methods \\ Patients and imaging datasets}

Retrospective analysis of publicly available datasets was performed after receiving approval from the Institutional Review Board at the Sungkyunkwan University, Korea. This study considered four independent datasets: two lung cancer, one kidney cancer, and one brain cancer datasets. We considered only primary cancers of each dataset in this study. Table 1 and supplementary material provide a detailed description of each dataset.

\section{Imaging differences at the whole organ level}

Each of the three organs that we studied might have a distinct intensity distribution due to inherent differences in an organ, as well as the differences in an imaging modality. A region of interest (ROI) was specified for each organ as a whole, and intensity histogram and the derived parameters of the histogram were compared. The training set (lung), test set2 (kidney), and test set3 (brain) were used for the analysis. Test set1 (lung) was not analyzed as it was the same organ as that in the training set (lung). Detailed segmentation methods are described in the supplementary methods. Histogram shapes were visually compared. Means and medians of the ROIs were also compared using two-sample t-tests. Within CT imaging (i.e., lung and kidney), the histogram shape was directly compared. Across imaging modalities (CT [lung and kidney] and MRI [brain]), we normalized the histograms using z-scores. The z-score normalization was one way to compare CT that has the standard Hounsfield unit (HU) with MRI that has an arbitrary unit. We only applied histogram normalization to compare histograms from different organs and did not apply the normalization when extracting the radiomics features. 
Table 1 Summary of all datasets

\begin{tabular}{lllll}
\hline & Training set & Test set1 & Test set2 & Test set3 \\
\hline Reference & NSCLC radiomics & TCIA lung CT diagnosis & TCGA-KIRC & CPTAC-GBM \\
Organ & Lung & Lung & Kidney & Brain \\
Modality & $C T$ & $C T$ & $C T$ & MRI \\
Number of patients & 422 (M: 290, F:132) & 61 (M: 31, F:30) & $48($ M:29, F: 19) & 43 (M: 31, F:12) \\
Age & 68.1 years (Avg.) & $\geq 65$ years: 41; & 65.3 years & 63 years \\
Stage & I-IIIb & I-IV years: 20 & I-IV & N/A \\
In-plane range $(\mathbf{m m})$ & $0.721-0.977$ (Avg. 0.976) & $0.586-0.953$ & $0.617-0.977$ (Avg. 0.785) & $0.469-1.016$ (Avg. 0. 598) \\
Avg Slice thickness $(\mathbf{m m})$ & 3.022 & (Avg. 0.736) & 4.708 & 1.575 \\
\hline
\end{tabular}

NSCLC non-small cell lung cancer, TCIA The Cancer Imaging Archive, TCGA-KIRC The Cancer Genome Atlas Kidney Renal Clear Cell Carcinoma, CPTAC-GBM Clinical Proteomic Tumor Analysis Consortium Glioblastoma Multiforme, Avg average, $M$ males, $F$ females

\section{ROI segmentation and feature extraction}

On axial CT and MRI images, tumors were segmented using in-house semi-automated software by a single expert (H.Y.L) [22]. A total of 143 radiomics features were computed from the native volume space over the ROIs $[8,22,23]$. The features were computed using a combination of open-source (PyRadiomics) and in-house codes in MATLAB (MathWorks, Inc., Natick, USA) [24]. Features that were unavailable in the PyRadiomics were locally implemented. The features consisted of 19 histogram-based, 11 shape-based, three fractal-based, 18 sigmoid function-based, 29 texture-based, and 63 filterbased features. Eight shape-based features were extracted using a three-dimensional (3D) ROI, and three shape-based features were two-dimensional (2D) [25]. The 2D shape-feature used the center slice of the 3D ROI. Three fractal-based features were the fractal dimension, fractal signature dissimilarity, and lacunarity $[26,27]$. Fractal dimension was computed using the boxcounting, and fractal signature dissimilarity (FSD) was computed using the blanket method. Eighteen sigmoid function-based features were computed to quantify the tumor margin properties [25]. Twenty-nine texturebased features were computed using gray-level cooccurrence matrix (GLCM, 256 bins, $n=22)$, gray-level size zone matrix (GLSZM, 32 bins, $n=2$ ), and neighborhood gray-tone difference matrix (NGTDM, $n=5)$ [20, 28-30]. GLCM features were computed in two ROI types using the whole ROI and sub-sampled ROI [23]. The filter-based features were computed using a 3D Laplacian of Gaussian (LoG) filter. The sigma values of the LoG filter were computed with $\sigma$ in 0.5 voxel increments (range, 0.5-3.5) [25]. A detailed description of the features is given in the supplement.

\section{Selection of features}

Radiomics analysis involved choosing a few features that can explain the intended target clinical variable well from many features. The feature selection was carried out in two steps in the training set. First, we chose stable features using the intra-class correlation (ICC) with IBM SPSS statistical software (IBM Corp., Armonk, USA). The stability of the 143 extracted features was assessed using the Reference Image Database to Evaluate Therapy Response (RIDER) dataset [31, 32]. We retained features with ICC values above 0.9 [33]. Second, we applied Cox - least absolute shrinkage selector operator (CoxLASSO) to select a few features related to survival from the selected features obtained from the first step. The hyperparameter of LASSO (i.e., regularization strength) was optimized using cross-validation. The MATLAB (MathWorks, Inc., Natick, USA) and "glmnet" R package (R Foundation for Statistical Computing, Vienna, Austria) were used for the Cox-LASSO. The CoxLASSO method was repeated 20 times, and only features that were selected more than ten times were chosen as the final selected features. In addition, we applied the Cox-LASSO feature selection to other datasets (test set1, 2 , and 3) to seek common features between the training set and each test set and test alternative models based on different datasets. The procedure was applied with a reduced threshold (10 times out of 40 ) due to convergence issues.

\section{Construction of radiomics score model and survival analysis}

We built a radiomics score related to survival using the selected features. Regression coefficients from the CoxLASSO were linearly combined with the feature values to yield a radiomics score. The radiomics score is a weighted (weights obtained from regression coefficients) sum of feature values [34, 35]. Each patient's radiomics score was computed. Patients were stratified into lowand high-risk groups using the median radiomics score as the cutoff [36]. Kaplan-Meier (KM) survival analysis was performed, and a log-rank test was used to compare 
the two risk groups using SPSS. The procedure was applied to the training set and validated in the three test sets. We developed the radiomics score model consisting of the selected features from the lung dataset and applied the model using the same features but refitted the coefficients to test it on the brain and kidney cases. The overall scheme of the study is given in Fig. 1.

\section{Results}

\section{Whole organ level imaging differences}

Based on the two-sample t-tests, mean values were significantly different between lung and kidney $(p<0.001$, $\left.3.43 \times 10^{-9}\right)$, lung and brain $\left(p=1.41 \times 10^{-8}\right)$, and kidney and brain $\left(p=9.53 \times 10^{-6}\right)$. Similarly, median values were significantly different between lung and kidney $\left(p=7.07 \times 10^{-19}\right)$, lung and brain $\left(p=1.22 \times 10^{-5}\right)$, and kidney and brain $\left(p=1.74 \times 10^{-8}\right)$. The box plots for mean and median values are shown in Fig. 2a.

The histogram shape was compared between CT images (lung and kidney) for a representative case (Fig. 2b). Intensity distribution did not overlap much between the two organs. Histogram was normalized to z-scores to make a comparison between CT and MRI modalities. All three distributions of the organs were centered at zero with distinct shapes for each organ (Fig. 2c).

\section{Selected features and radiomics score}

In the first selection step using the ICC threshold, 97 features were selected as stable features. The selected 97 features were used in the second feature selection step using Cox-LASSO. Finally, 7 features were selected from the training set. The final selected features were as follows: histogram-based features (2.5 percentile and 97.5 percentile); shape-based features (sphericity, maximum 3D diameter, and roundness factor [2D]); and texture-based feature (an informational measure of correlation [sub-sampled GLCM] and size-zone variability). One of the features, 2.5 percentile, is a well-known lungspecific feature [37]. We computed another radiomics score with the 2.5 percentile feature removed using the six features, to see if removing lung-specific features would lead to better generalization to other organs (i.e., kidney and brain). The ensuing survival analysis was performed using two radiomics scores.

\section{Survival analysis using all the selected features}

Survival analysis was performed, and patients were divided into high- and low-risk groups using the median radiomics score as the cut-off. The radiomics score was computed using all seven selected features from the training set. In the training set (lung), two risk groups were well-stratified using the log-rank test $(p<0.001)$ (Fig. 3a). There was a similar significant difference $(p=$ 0.012) in survival between the two risk groups in the test set1 (lung) using the radiomics score computed from the test set1 (Fig. 3b). However, the results from test set2 and test set3 were different. The test set2 (kidney) did not show any significant differences in survival between the two risk groups, and the log-rank test was not statistically significant $(p=0.713)$ (Fig. 3c). In the test set3 (brain), differences in survival between the two risk groups were visually noticeable in the plot, but the difference was not statistically significant $(p=0.105)$ (Fig. $3 \mathrm{~d})$. In summary, the radiomics model trained from the

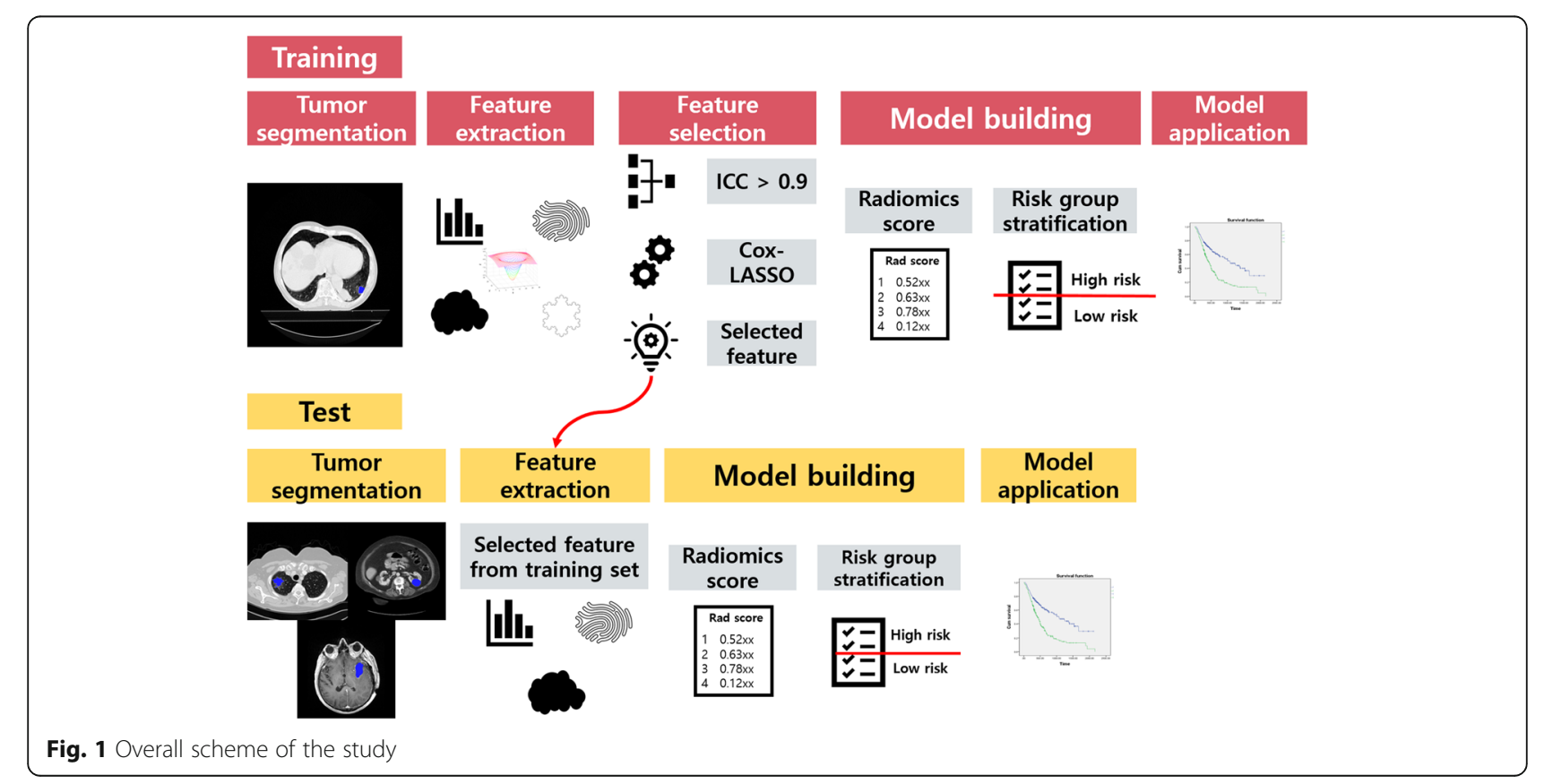



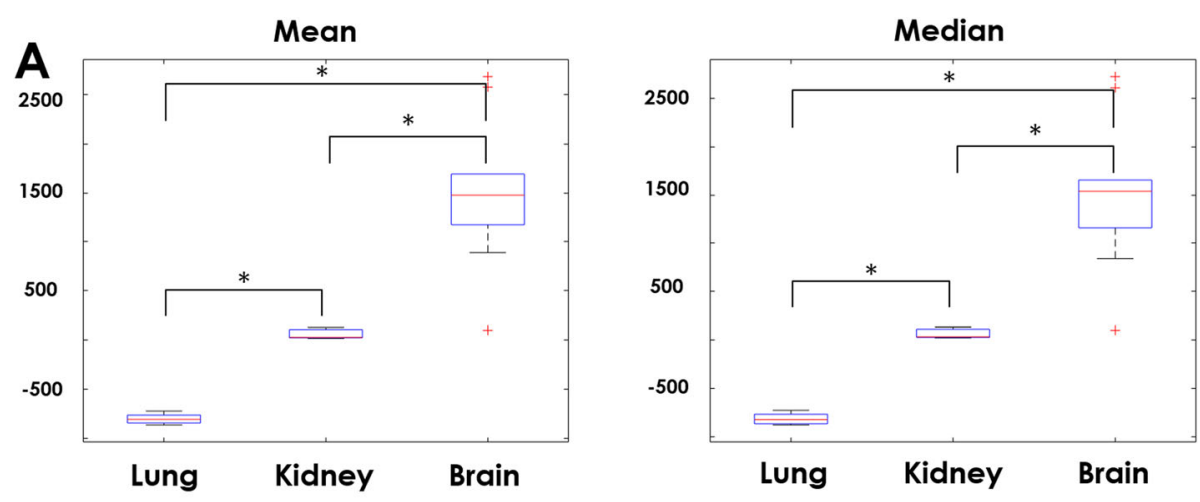

B 0.04
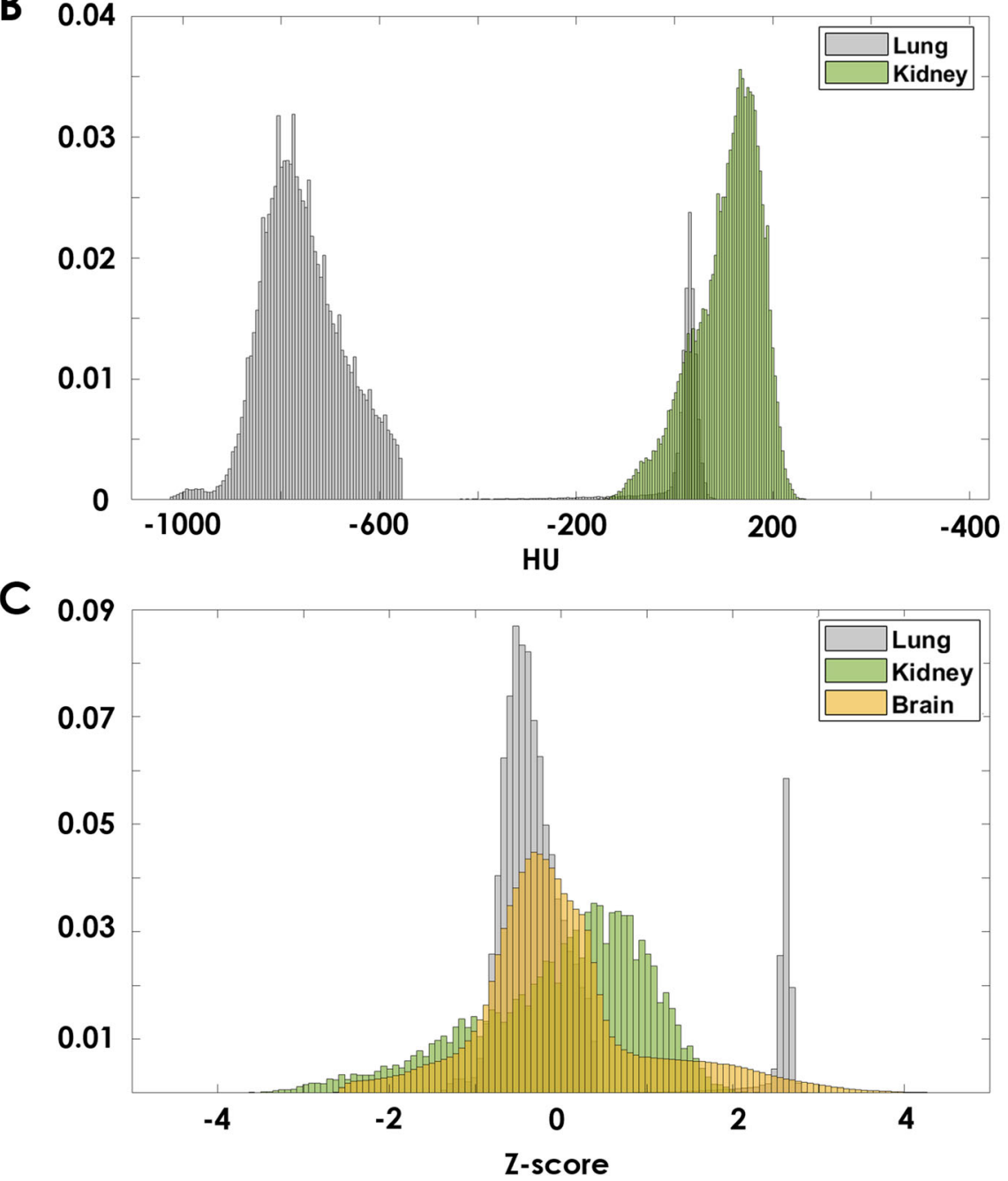

Fig. 2 Imaging differences at the whole-organ level. a Box plots for mean and median; b comparison of histogram within CT for lung (gray color) and kidney (green color); and c comparison of normalized histogram across modalities for lung (gray color), kidney (green color), and brain (yellow color)

lung data was only useful for stratifying survival in the other lung data, and not in other organs like the kidney and brain. Additional results of using kidney and brain datasets to build survival models were shown in the Supplement.
Survival analysis without the lung-specific feature

The same survival analysis was performed using the radiomics score without the lung-specific 2.5 percentile feature. The results were largely similar to those using the radiomics score for all the features. In the training 
$\neg \leq$ Median

$\neg>$ Median

$+\leq$ Median-censored

$+>$ Median-censored
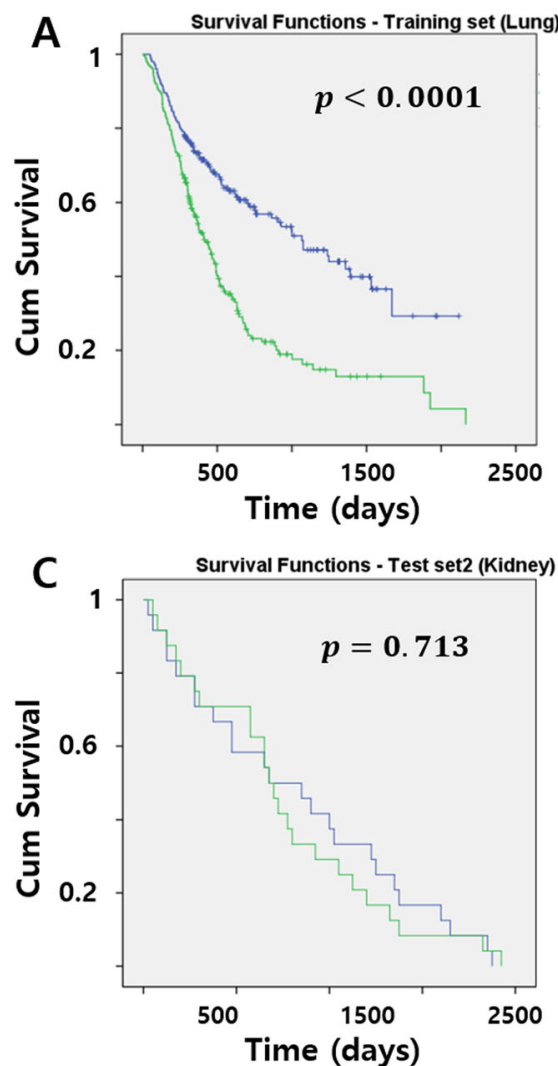
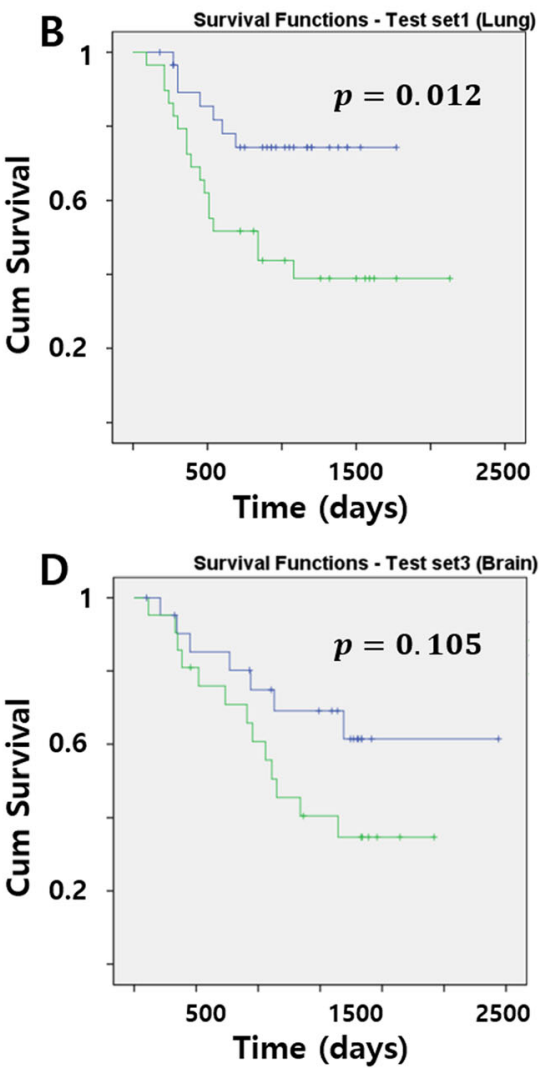

Fig. 3 The Kaplan-Meier plots using the radiomics score of all the selected features of (a) training set (top left), (b) test set1 (top right), (c) test set2 (bottom left), and (d) test set3 (bottom right). The blue line is the low-risk group ( $\leq$ Median), and the green line is the high-risk group (> Median)

set (lung), the two risk groups were well-stratified using the log-rank test $(p<0.001)$ (Fig. 4a). There was a similar significant difference $(p=0.033)$ in the survival between the two risk groups in test setl (lung) using the radiomics score computed from the test set1 (Fig. 4b). However, the results from test set2 and test set3 were different. The test set2 (kidney) showed visual differences in the survival plot but did not show any significant differences $(p=0.062)$ (Fig. $4 c)$. In the test set3 (brain), differences in survival between the two risk groups were visually noticeable in the plot, but there was no statistical significance $(p=0.356)$ (Fig. $4 d$ ). In summary, the radiomics model trained from the lung data was only effective in stratifying survival in the other lung data, and not in other organs like the kidney and brain.

\section{Common features between the training set and test sets}

We observed that the radiomics model trained from the lung data was only effective in stratifying survival in the other lung data, and not in other organs like the kidney and brain. This might imply that features reflecting survival could be different for different organs. Thus, we performed the same feature selection approach on all four datasets and compared the features selected among different datasets across three organs. In the test set1, variance, standard deviation, 50 percentile (histogram), homogeneity (sub-sampled GLCM), roundness factor (2D shape), and FSD (fractal) were selected. In test set2, energy, difference entropy (sub-sampled GLCM), and contrast (NGTDM) were selected. In the last test set3, informational measures of correlation (sub-sampled GLCM) and coarseness (NGTDM) were selected. The roundness factor (2D shape) was a common feature between the training set and test set1. No common features were found between the training set and test set2. The informational measure of correlation (sub-sampled GLCM) was a common feature between the training set and test set3. There were no common features between the training set and various test sets, but there was a category of features that was common. All datasets had at 

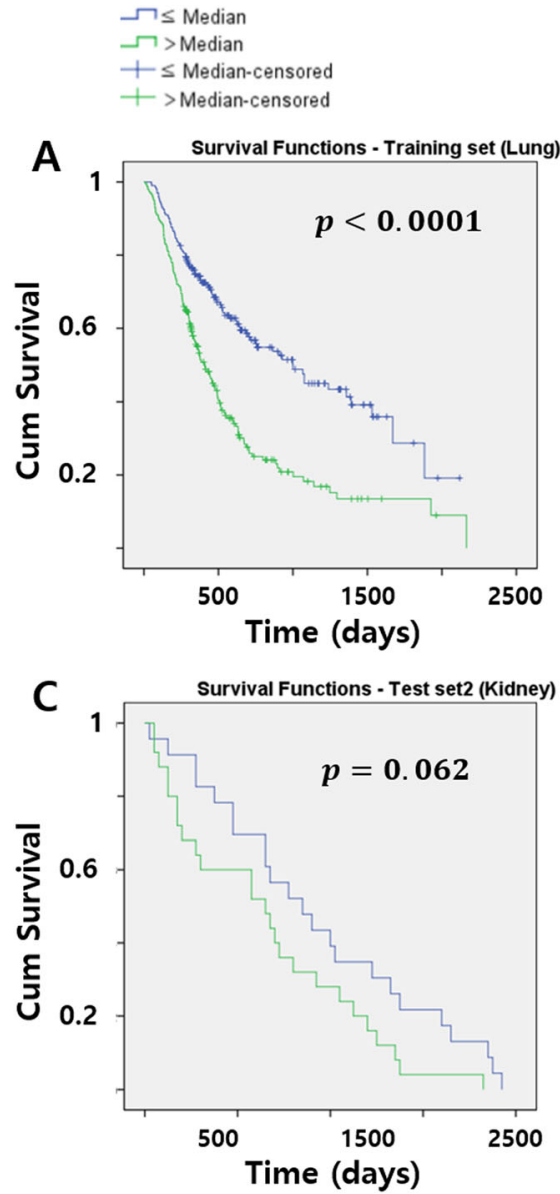
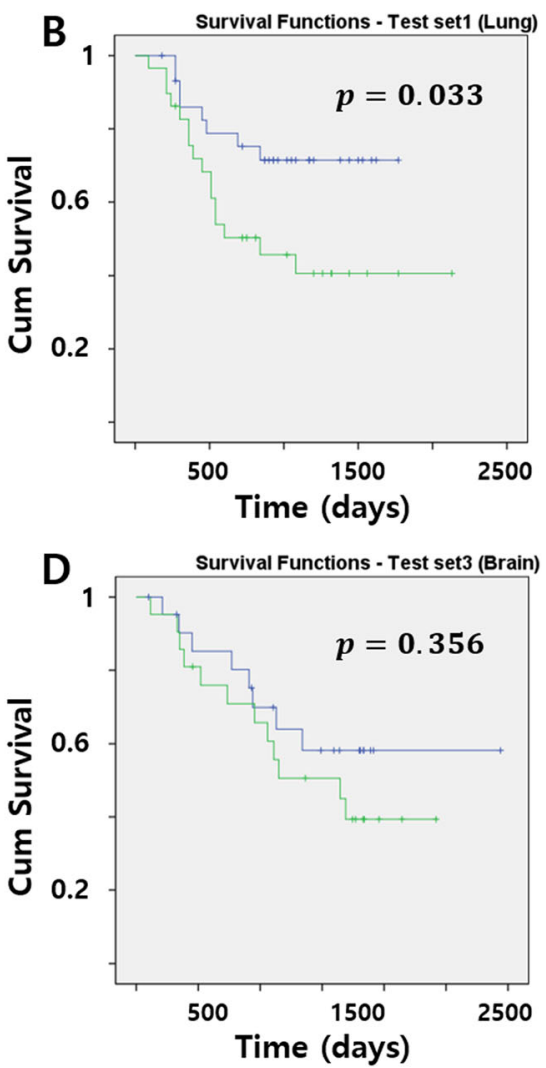

Fig. 4 The Kaplan-Meier plots using radiomics score with the lung-specific feature removed of ((a) training set (top left), (b) test set1 (top right), (c) test set2 (bottom left), and (d) test set3 (bottom right). The blue line is the low-risk group ( $\leq$ Median), and the green line is the high-risk group (> Median)

least one GLCM feature as the chosen feature denoting the importance of texture feature in radiomics. Full details are given in Table 2.

\section{Discussion}

We confirmed that there were obvious differences in imaging at the organ level for the three organs by comparing their intensity histograms. The radiomics score and the associated features derived from the training set (lung) were effective for the test set1 (the other lung) in stratifying survival, but not for the kidney and brain (test set2 and set3). A similar trend was observed even when we removed the lung-specific feature (2.5 percentile) from the radiomics score.

Many radiomics studies have discovered features related to the tumor microenvironment and the associated macroscale features. In particular, features in the texture category have been commonly selected for various radiomics studies. In one study, GLCM-based features contributed to the discovery of radiomics signatures predicting immune microenvironment and patient outcomes [15]. Texture-based features were identified as important features to predict immune responses [19]. Texture-based features, unlike the histogram and shapebased features, incorporate the neighborhood information and hence are better suited to describe the tumor microenvironment.

We explored whether features trained in a specific organ can be applied to other organs. In Fig. 2, we confirmed that different organs have distinct differences in intensity distribution. Figure $2 \mathrm{~b}$ shows that a lung CT scan is quite different from that of the kidney due to the unique environment of the lungs, which includes a large proportion of air. This distinct information for each organ makes finding a universal feature set difficult across different organs. The features derived from the training set (lung) were not useful in stratifying survival in the kidney and brain (test set2 and set3) datasets. To correct for the differences among organs, additional experiments to remove the feature related to air components (2.5 percentile) were performed. This was to test whether removing the lung-specific feature 
Table 2 Results of feature selection for each dataset

\begin{tabular}{|c|c|c|c|}
\hline Dataset & Feature category & Feature name & Repetition times \\
\hline \multirow[t]{7}{*}{ Training (7 features) } & \multirow[t]{2}{*}{ Histogram-based } & 2.5 percentile & 20 \\
\hline & & 97.5 percentile & 20 \\
\hline & \multirow[t]{2}{*}{ Shape-based (3D) } & Sphericity & 20 \\
\hline & & Max 3D diameter & 20 \\
\hline & Shape-based (2D) & Roundness factor & 20 \\
\hline & \multirow[t]{2}{*}{ Texture-based } & Informational Measure of Correlation, subsampled GLCM & 17 \\
\hline & & Size zone variability & 10 \\
\hline \multirow{6}{*}{$\begin{array}{l}\text { Test set } 1 \\
\text { (6 features) }\end{array}$} & \multirow[t]{2}{*}{ Histogram-based } & Variance & 20 \\
\hline & & 50 Percentile & 20 \\
\hline & Texture-based & Homogeneity, subsampled GLCM & 15 \\
\hline & Shape-based (2D) & Roundness factor & 9 \\
\hline & Fractal-based & FSD & 4 \\
\hline & Histogram-based & Standard deviation & 1 \\
\hline \multirow{3}{*}{$\begin{array}{l}\text { Test set2 } \\
\text { (3 features) }\end{array}$} & \multirow[t]{3}{*}{ Texture-based } & Energy, subsampled GLCM & 1 \\
\hline & & Contrast, NGTDM & 1 \\
\hline & & Difference entropy, subsampled GLCM & 1 \\
\hline \multirow{2}{*}{$\begin{array}{l}\text { Test set } 3 \\
\text { ( } 2 \text { features) }\end{array}$} & \multirow[t]{2}{*}{ Texture-based } & Informational Measure of Correlation, subsampled GLCM & 19 \\
\hline & & Coarseness, NGTDM & 3 \\
\hline
\end{tabular}

Bold font indicates the features commonly selected between the training set and given test sets. $2 D$ two-dimensional, $3 D$ three-dimensional, $G L C M$ gray-level cooccurrence matrix, FSD fractal signature dissimilarity, NGTDM neighborhood gray-tone difference matrix

could lead to better generalizability to the other two organs. However, the results were somewhat similar. Despite the lung-specific feature removed, the features derived from the training data could not be applied to the other two organs. This observation indicated that radiomics features of a given organ from a tumor ROI reflected a specific tumor microenvironment and the associated macroscale features. Therefore, applying the radiomics feature derived from one organ to other organs requires careful consideration of specific properties of the target organ.

Both training set and test set 1 were from lung-cancer databases, but there was only one common feature selected (Table 2, roundness factor). This might imply that circularity is more important in lung cancer compared to other organs. The data of the training set came from various types of lung cancer patients: large cell, squamous cell carcinoma, and adenocarcinoma. However, the entire data of test set1 were derived from patients with adenocarcinoma. Thus, this difference in subtype composition could have led to different features being selected. Between the training set and test set3, one common feature was selected, the informational measure of correlation. This confirmed that lung cancer and glioblastoma were both associated with the secondary measure of homogeneity.

To the best of our knowledge, no radiomics study has attempted to find common features in different organs systematically. Some studies explored the concept of universal radiomics features, but it was their secondary objective, not the main one. One study adopted a procedure similar to ours. The study built radiomics models using lung cancer data and applied them to H\&N cancer data. They showed that the same radiomics model was effective for predicting survival in two organs, but the results could be invalid because their feature selection approach was biased [20]. Another study attempted to explore the feasibility of applying the same set of radiomics features [21]. However, the goal was not to find common features but to compare the performance of $2 \mathrm{D}$ and $3 \mathrm{D}$ radiomics in intrahepatic cholangiocarcinoma, osteosarcoma, and pancreatic neuroendocrine tumors.

Although common features were elusive, we reconfirmed that texture features could be a common category of features for the three organs. This finding is largely in line with many radiomics studies emphasizing the importance of texture features to capture tumor heterogeneity. Active research is ongoing to propose new texture features, some of which could be applied to many organs.

Recently, we witnessed increased adoption of deep learning (DL) in imaging analysis that led to improved performances in many organs [38-40]. DL approaches use more features (even in tens of thousands) than radiomics approaches (hundreds to thousands) in a data-driven fashion. The intermediate layers of the DL network are commonly used for data-driven feature 
representation. As there are more features to choose from, there could be a better chance of finding common features between different organs. DL approaches are inherently multi-scale, and thus common features might be found in small-scale low-level features.

Our study has some limitations. The sample size of the test sets was rather small $(n<100)$; thus, further studies using larger samples are necessary to validate the results of our study. We explored radiomics models for survival analysis and did not consider other clinical outcomes, such as tumor grading, that need to be explored in the future. Our results were limited to three organs (lung, kidney, and brain) and two imaging modalities (CT and MRI). Therefore, future studies may extend this approach to more organs and modalities. Our model from the training set had three subtypes of NSCLC and thus might not have the transfer capability to one specific subtype of NSCLC. Exploring the subtype-specific transferability is an important future research direction. Finally, there is no agreed standard method to extract and select radiomics features, which makes tight controlling of the experiments difficult. Although our method is a widely used one $[23,36,41]$, our results were specific to the adopted methods and thus should be interpreted with care.

\section{Conclusion}

Overall, we suggest that radiomics score models for survival were mostly specific for a given organ. This was confirmed by the absence of any common features being identified between the training set and various test sets. However, we noticed that one category of features (texture category) was common in all three organs. In sum, applying radiomics score models to other organs would require careful consideration of organ-specific properties. Still, caution should be taken when constructing models with radiomics features because we cannot exclude the possibility to construct a generally applicable model for various organs given an optimal model.

\section{Abbreviations}

NSCLC: Non-small cell lung cancer; HU: Hounsfield unit; LoG: Laplacian of Gaussian; GLCM: Gray-level co-occurrence matrix; GLSZM: Gray-level size zone matrix; NGTDM: Neighborhood gray-tone difference matrix; FSD: Fractal signature dissimilarity; Cox-LASSO: Cox - least absolute shrinkage selector operator; ICC: Intra-class correlation; KM: Kaplan-Meier

\section{Supplementary Information}

The online version contains supplementary material available at https://doi. org/10.1186/s40644-021-00400-y.

Additional file 1: Supplementary Table 1. Definition of extracted radiomics features. Supplementary Methods. Patients and imaging datasets. Imaging differences at the organ level. Supplementary

Results. Fig. S1. The Kaplan-Meier plots using the radiomics score based on test set2 (kidney). Fig. S2. The Kaplan-Meier plots using the radiomics score based on test set2 (brain).

\section{Acknowledgments}

This research was supported by the National Research Foundation (NRF2020R1F1A1068226, NRF-2020M3E5D2A01084892), Institute for Basic Science (IBS-R015-D1), Ministry of Science and ICT (IITP-2020-2018-0-01798), IITP grant funded by the AI Graduate School Support Program (2019-0-00421), and ICT Creative Consilience program (IITP-2020-0-01821).

\section{Authors' contributions}

Seung-Hak Lee conceived, designed, and performed the experiments. Hwanho Cho aided the experiments and analyzed the data. Ho Yun Lee conceived and designed the experiments. She reviewed drafts of the paper and approved the final draft. She is the guarantor of this study. Hyunjin Park conceived and designed the experiments. He reviewed drafts of the paper and approved the final draft

\section{Funding}

This research was supported by the National Research Foundation (NRF2020R1F1A1068226, NRF-2020M3E5D2A01084892), Institute for Basic Science (IBS-R015-D1), Ministry of Science and ICT (IITP-2020-2018-0-01798), IITP grant funded by the Al Graduate School Support Program (2019-0-00421), and ICT Creative Consilience program (IITP-2020-0-01821).

Availability of data and materials

The data and material are available through one of the corresponding authors (Dr. Ho Yun Lee).

\section{Declarations}

Ethics approval and consent to participate

Institutional review board (IRB) approvals from Samsung Medical Center (SMC) and Sungkyunkwan University were obtained for this retrospective study with waivers of informed consent.

Consent for publication

All authors gave consent for publication.

Competing interests

None.

\section{Author details}

${ }^{1}$ Departement of Electronic Electrical and Computer Engineering, Sungkyunkwan University, Suwon 16419, South Korea. ${ }^{2}$ Center for Neuroscience Imaging Research, Institute for Basic Science (IBS), Suwon 16419, South Korea. ${ }^{3}$ Core Research \& Development Center Korea University Ansan Hospital, Ansan 15355, South Korea. ${ }^{4}$ Department of Radiology and Center for Imaging Science, Samsung Medical Center, Sungkyunkwan University School of Medicine, 81 Irwon-Ro, Gangnam-Gu, Seoul 06351, South Korea. ${ }^{5}$ Department of Health Sciences and Technology, SAIHST, Sungkyunkwan University, Seoul 06351, South Korea. '5chool of Electronic and Electrical Engineering, Center for Neuroscience Imaging Research, Sungkyunkwan University, Suwon 16419, South Korea.

Received: 23 April 2020 Accepted: 26 March 2021

Published online: 07 April 2021

\section{References}

1. Giardino A, Gupta S, Olson E, Sepulveda K, Lenchik L, Ivanidze J, et al. Role of Imaging in the Era of Precision Medicine [Internet]. Acad Radiol Elsevier Inc. 2017, 639:-49 Available from: http://dx.doi.org/10.1016/j.acra. 2016.11.021.

2. Wagenaar HC, Trimbos JBMZ, Postema S, Anastasopoulou A, Van Der Geest RJ, Reiber JHC, et al. Tumor diameter and volume assessed by magnetic resonance imaging in the prediction of outcome for invasive cervical cancer. Gynecol Oncol. 2001;82(3):474-82. https://doi.org/10.1006/gyno.2 001.6267.

3. Grove O, Berglund AE, Schabath MB, Aerts HJWL, Dekker A, Wang H, et al. Quantitative computed tomographic descriptors associate tumor shape complexity and intratumor heterogeneity with prognosis in lung adenocarcinoma. PLoS One. 2015;10:1-14.

4. Chong Y, Kim J-H, Lee HY, Ahn YC, Lee KS, Ahn M-J, et al. Quantitative CT Variables Enabling Response Prediction in Neoadjuvant Therapy with EGFR- 
TKIs: Are They Different from Those in Neoadjuvant Concurrent Chemoradiotherapy? PLoS One [Internet]. 2014;9:e88598 Available from: http://dx.plos.org/10.1371/journal.pone.0088598.

5. Gillies RJ, Kinahan PE, Hricak H. Radiomics: images are more than pictures, they are data. Radiology. 2016;278(2):563-77. https://doi.org/10.1148/radiol.2 015151169.

6. Aerts HJWL. The potential of radiomic-based phenotyping in precisionmedicine a review. JAMA Oncol. 2016;2(12):1636-42. https://doi. org/10.1001/jamaoncol.2016.2631

7. Zhang B, Tian J, Dong D, Gu D, Dong Y, Zhang L, et al. Radiomics features of multiparametric MRI as novel prognostic factors in advanced nasopharyngeal carcinoma. Clin Cancer Res. 2017;23(15):4259-69. https:// doi.org/10.1158/1078-0432.CCR-16-2910.

8. Lee SW, Park H, Lee HY, Sohn I, Lee SH, Kang J, et al. Deciphering Clinicoradiologic phenotype for Thymidylate synthase expression status in patients with advanced lung adenocarcinoma using a Radiomics approach. Sci Rep Springer US. 2018;8:1-10

9. Kolossváry M, Karády J, Szilveszter B, Kitslaar P, Hoffmann U, Merkely B, et al. Radiomic features are superior to conventional quantitative computed tomographic metrics to identify coronary plaques with napkin-ring sign. Circ Cardiovasc Imaging. 2017;10:1-9.

10. Coroller TP, Agrawal V, Narayan V, Hou Y, Grossmann P, Lee SW, et al. Radiomic phenotype features predict pathological response in non-small cell Radiomic predicts pathological response lung cancer. Radiother Oncol [internet]. Elsevier Ireland Ltd. 2016;1 19(3):480-6. Available from: http://dx. doi.org/10.1016/j.radonc.2016.04.004.

11. Iqbal S, Khan MUG, Saba T, Rehman A. Computer-assisted brain tumor type discrimination using magnetic resonance imaging features. Biomed Eng Lett The Korean Society of Medical and Biological Engineering. 2018:5-28.

12. Yip SSF, Aerts HJWL. Applications and limitations of radiomics. Phys Med Biol IOP Publishing. 2016;61 (13):R150-66. https://doi.org/10.1088/0031-9155/ 61/13/R150.

13. Yoon HJ, Kang J, Park H, Sohn I, Lee SH, Lee HY. Deciphering the tumor microenvironment through radiomics in non-small cell lung cancer: correlation with immune profiles. PLoS One. 2020;15:1-13.

14. Sun R, Limkin EJ, Vakalopoulou M, Dercle L, Champiat S, Han SR, et al. A radiomics approach to assess tumour-infiltrating CD8 cells and response to anti-PD-1 or anti-PD-L1 immunotherapy: an imaging biomarker, retrospective multicohort study. Lancet Oncol. 2018;19(9):1180-91. https:// doi.org/10.1016/S1470-2045(18)30413-3.

15. Tang C, Hobbs B, Amer A, Li X, Behrens C, Canales JR, et al. Development of an immune-pathology informed Radiomics model for non-small cell lung Cancer. Sci rep [internet]. Springer US. 2018;8(1):1-9. Available from: http:// dx.doi.org/10.1038/s41598-018-20471-5.

16. Roma-Rodrigues C, Mendes R, Baptista PV, Fernandes AR. Targeting tumor microenvironment for cancer therapy. Int J Mol Sci. 2019.

17. Binnewies M, Roberts EW, Kersten K, Chan V, Fearon DF, Merad M, et al. Understanding the tumor immune microenvironment (TIME) for effective therapy. Nat med [internet] Springer US. 2018;24(5):541-50. Available from: http://dx.doi.org/10.1038/s41591-018-0014-x.

18. Lee G, Lee HY, Park H, Schiebler ML, van Beek EJR, Ohno Y, et al. Radiomics and its emerging role in lung cancer research, imaging biomarkers and clinical management: State of the art. Eur J Radiol. 2017:297-307.

19. Trebeschi S, Drago SG, Birkbak NJ, Kurilova I. Predicting response to cancer immunotherapy using noninvasive radiomic biomarkers. Ann Oncol. 2019; 30(6):998-1004. https://doi.org/10.1093/annonc/mdz108.

20. Aerts HJWL, Velazquez ER, Leijenaar RT, Parmar C, Grossmann P, Carvalho S, et al. Decoding tumour phenotype by noninvasive imaging using a quantitative radiomics approach. Nat Commun. 2014;5(1):4006. https://doi. org/10.1038/ncomms5006

21. Xu L, Yang $P$, Yen EA, Wan Y, Jiang $Y$, Cao Z, et al. A multi-organ cancer study of the classification performance using $2 \mathrm{D}$ and $3 \mathrm{D}$ image features in radiomics analysis. Phys Med Biol IOP Publishing. 2019;64.

22. Song SH, Park H, Lee G, Lee HY, Sohn I, Kim HS, et al. Imaging Phenotyping using Radiomics to predict micropapillary pattern within lung adenocarcinoma. J Thorac Oncol [internet]. Elsevier Inc. 2017;12(4):624-32. Available from: $h$ ttp://dx.doi.org/10.1016/j.jtho.2016.11.2230.

23. Lee $\mathrm{S}-\mathrm{H}$, Cho H, Lee HY, Park H. Clinical impact of variability on $\mathrm{CT}$ radiomics and suggestions for suitable feature selection: a focus on lung cancer. Cancer Imaging Cancer Imaging. 2019;19:1-12.
24. Van Griethuysen JJM, Fedorov A, Parmar C, Hosny A, Aucoin N, Narayan V, et al. Computational radiomics system to decode the radiographic phenotype. Cancer Res. 2017;77(21):e104-7. https://doi.org/10.1158/0008-54 72.CAN-17-0339.

25. Aerts HJWL, Grossmann P, Tan Y, Oxnard GG, Rizvi N, Schwartz LH, et al. Defining a Radiomic response phenotype: a pilot study using targeted therapy in NSCLC. Sci Rep Nature Publishing Group. 2016;6.

26. Wang $C$, Subashi $E$, Yin FF, Chang Z. Dynamic fractal signature dissimilarity analysis for therapeutic response assessment using dynamic contrast-enhanced MRI. Med Phys. 2016;43(3):1335-47. https://doi.org/1 $0.1118 / 1.4941739$

27. Lennon FE, Cianci GC, Cipriani NA, Hensing TA, Zhang HJ, Chen C-T, et al. lung cancer-a fractal viewpoint. Nat rev Clin Oncol Nat Publ Group. 2015; 12:664-75.

28. Tixier F, Le Rest CC, Hatt M, Albarghach N, Pradier O, Metges J-P, et al. Intratumor Heterogeneity Characterized by Textural Features on Baseline 18F-FDG PET Images Predicts Response to Concomitant Radiochemotherapy in Esophageal Cancer. J Nucl Med [Internet]. 2011;52: 369-78 Available from: http://jnm.snmjournals.org/cgi/doi/10.2967/jnumed. 110.082404

29. Haralick RM, Shanmugam K. Textural features for image classification. IEEE Trans Syst Man Cybern. 1973;SMC-3(6):610-21. https://doi.org/10.1109/ TSMC.1973.4309314.

30. Niu L, Qian M, Yang W, Meng L, Xiao Y, Wong KKL, et al. Surface roughness detection of arteries via texture analysis of ultrasound images for early diagnosis of atherosclerosis. PLoS One. 2013;8(10):e76880. https://doi.org/1 0.1371/journal.pone.0076880

31. Zhao B, James LP, Moskowitz CS, Guo P, Ginsberg MS, Lefkowitz RA, et al. Evaluating variability in tumor measurements from same-day repeat $C T$ scans of patients with non-small cell lung cancer. Radiology. 2009;252(1): 263-72. https://doi.org/10.1148/radiol.2522081593.

32. Clark K, Vendt B, Smith K, Freymann J, Kirby J, Koppel P, et al. The cancer imaging archive (TCIA): maintaining and operating a public information repository. J Digit Imaging. 2013;26(6):1045-57. https://doi.org/10.1007/s102 78-013-9622-7.

33. Liu Y, Zhang Y, Cheng R, Liu S, Qu F, Yin X, et al. Radiomics analysis of apparent diffusion coefficient in cervical cancer: a preliminary study on histological grade evaluation. J Magn Reson Imaging. 2018:1-11.

34. Huang Y, Liu Z, He L, Chen X, Pan D, Ma Z, et al. Radiomics signature : a potential biomarker for the prediction of disease-free survival in early-stage (I or II) non—small cell lung Cancer. Radiology. 2016;000:1-11.

35. Zheng BH, Liu LZ, Zhang ZZ, Shi JY, Dong LQ, Tian LY, et al. Radiomics score: a potential prognostic imaging feature for postoperative survival of solitary HCC patients. BMC Cancer BMC Cancer. 2018;18:1-12.

36. Park H, Kim J, Sj C, Sh L, Hy L, Park H. Predicting survival using pretreatment CT for patients with hepatocellular carcinoma treated with Transarterial chemoembolization: comparison of models using Radiomics. Am J Roentgenol. 2018:1-9.

37. Ella A, BHG K. Cardiopulmonary Imaging: Lippincott Williams \& Wilkins; 2004.

38. Hosny A, Parmar C, Coroller TP, Grossmann P, Zeleznik R, Kumar A, et al. Deep learning for lung cancer prognostication: a retrospective multi-cohort radiomics study. PLoS Med. 2018;15:1-25.

39. Lou B, Doken S, Zhuang T, Wingerter D, Gidwani M, Mistry N, et al. Articles An image-based deep learning framework for individualising radiotherapy dose : a retrospective analysis of outcome prediction. Lancet Digit Heal [Internet]. 2019;1:e136-47 The Author(s). Published by Elsevier Ltd. This is an Open Access article under the CC BY-NC-ND 4.0 license. Available from: http://dx.doi.org/10.1016/S2589-7500(19)30058-5.

40. Yonekura A, Kawanaka H, Prasath VBS, Aronow BJ, Takase H. Automatic disease stage classification of glioblastoma multiforme histopathological images using deep convolutional neural network. Biomed Eng Lett [internet] The Korean Society of Medical and Biological Engineering; 2018;8; 321-327. Available from: https://doi.org/10.1007/s13534-018-0077-0, 2018.

41. Kim KH, Kim J, Park H, Kim H, hak LS, Sohn I, et al. parallel comparison and combining effect of radiomic and emerging genomic data for prognostic stratification of non-small cell lung carcinoma patients. Thorac Cancer. 2020; 11(9):2542-51. https://doi.org/10.1111/1759-7714.13568.

\section{Publisher's Note}

Springer Nature remains neutral with regard to jurisdictional claims in published maps and institutional affiliations. 